\title{
Mercuric chloride poisoning: Oesophagectomy as treatment?
}

\author{
Sara Al Awad ${ }^{1 *}$, Laurent Gruner ${ }^{2,3}$ and Olivier Monneuse M,5 $^{4,5}$ \\ ${ }^{1}$ Post-surgical residency, Lyon, France \\ ${ }^{2}$ Senior consultant, General Surgery, Lyon, France \\ ${ }^{3}$ Senior Registrar, General Surgery, University Hospital Edouard Herriot, France \\ ${ }^{4}$ Professor, General surgery and Trauma Surgeon, Head, Trauma Surgery Department, Lyon, France \\ ${ }^{5}$ Head, Surgical Department, University Hospital Edouard Herriot, France
}

\begin{abstract}
Background: Mercury is one of the most dangerous heavy metals if repeatedly ingested. It existed for medicinal purposes such as thermometers, for disinfection in Arabic medicine and in the consumption of fish in Japan. It exists in several forms and the medical care required for these various forms depends on the mode of intake, the dose and more specifically the pharmacokinetics of each compound. Ingestion of Mercuric chloride is a very rare and medically challenging dilemma as it requires an early multi-departmental approach to the management of the patient.
\end{abstract}

Clinical Presentation: A 32-year-old patient ingested $2 \mathrm{~g}$ of mercuric chloride for suicidal reasons. Initially presenting to the emergency department with nausea and vomiting admitting she had ingested this compound arose our vigilance with immediate introduction of the chelating agent Dimercaprol and she underwent a series of endoscopies as well as computed tomography (CT) scans before her case deteriorated and she was taken to the operating theatre on day 23 for a total esophagogastrectomy, oesophagostomy and jejunostomy for enteral alimentation.

Understanding the sequence of events and the dose of ingested mercuric chloride leads us to a certain degree of assurance that a surgical intervention was required earlier in the course of treatment. The early administration of the chelating agent which has great affinity for mercury compounds helps excrete the mercury safely, the oropharyngeal examination in the initial phase is of great importance as with its corrosive function it may cause an inflammatory stenosis response and/or an eventual intubation. Topographies and endoscopies are also critical in deciding the subsequent steps. Due to the fact that patients develop acute renal failure dialysis becomes a priority as well.

Conclusion: What is of importance to us is the multidisciplinary approach to these specific and rare group of patients. The medical care should be meticulously organised in a systematic manner in order for the right decision to be carried out at the right time. In this case of mercuric chloride poisoning an earlier oesophagectomy would have definitely avoided the ongoing necrotic process and would have provided the patient with an earlier reconstructive surgery.

Abbreviations: CT: computed tomography; BAL: British Anti Lewisite; DMSA: Dimercaptopropanesulfonate.

\section{Background}

Mercury or quicksilver as it was once called is a toxic heavy metal with dangerous side effects if ingested. Mercury is the third most toxic element on the planet, according to the US Government Agency for Toxic Substances and Disease Registry [1]. It exists in three forms: elemental (metallic), inorganic and organic mercury compounds. Inorganic mercury compounds are water soluble with a bioavailability of $7 \%$ to $15 \%$ after ingestion, they are also considered harmful to the gastrointestinal system as they have shown corrosive effects. However, elemental mercury is lipid soluble and is poorly absorbed in the gastrointestinal tract if ingested with a bioavailability at $0.01 \%$. Elemental mercury compounds have the capacity to cross the blood-brain barrier and placental barrier unlike inorganic mercury compounds [1-5].

Used once to treat syphilis between 1955 and 1965 the toxicity of this metal became obviously clear to physicians 50 years ago. Chelating agents such as Dimercaprol also known as British Anti Lewisite (BAL) have been used to treat inorganic mercury poisoning and minimise the risk of the ongoing necrotic process of the digestive tube after ingestion.

This article explains the effect of ingested mercuric chloride in one of our patients as she attempted to commit suicide and the different stages, factors as well as elements we were faced with in order to save her life.

Mercuric chloride or mercury chloride is an inorganic mercury compound that has a biphasic half-life of (2-4 days and 15-45 days). Approximately 1 to $4 \mathrm{~g}$ of mercuric chloride is fatal in adults [6]. Its treatment includes gastric lavage, chelating agent BAL and in case of acute tubular necrosis dialysis will be necessary as explained by Poupona, [2].

\section{Case presentation}

A patient of 32 years old with past medical history of depression, anxiety and a road traffic accident causing traumatic haemoperitoneum

*Correspondence to: Sara Al Awad, Post-surgical residency, Lyon, France, Tel: 0033-6-34075146; E-mail: sara_alawad@hotmail.com

Received: May 15, 2020; Accepted: May 21, 2020; Published: May 26, 2020 
and transverse process fracture of L4 and L5 in 2015, presents to the emergency department of our hospital complaining of sudden severe abdominal pain with hematemesis after admitting swallowing $2 \mathrm{~g}$ of mercuric chloride bought from the internet and fitted into 2 painkiller pills for suicidal reasons. Before arriving at the hospital, the paramedics contacted the poison control center for the management of the chelating agent BAL (British Anti-Lewisite). Upon arrival, she is sent immediately to the intensive care unit where she had a CT scan that showed the presence of mercury along with mucosal wall thickening of the lower two-thirds of the oesophagus but without oesophageal perforation. It was difficult to assess the oesophageal mucosa on CT due to mercury artefact. Followed by a gastroscopy under general anaesthesia which showed mucosal erosions and ulcerations in her lower two-thirds of the oesophagus as well as the stomach cardia and fundus classified as type IIIb and Grade III respectively. She was kept in the intensive care unit for another endoscopy 12 hours later. The maximum concentration of mercury on the second day upon admission was measured as high as 4940,6ug/L [7,8].

The patient rapidly presented acute renal failure in the following days which required her transfer to reanimation and a continuous dialysis for 72 hours with a slow recovery of diuresis. She was also re-scanned a few times showing the stability of the oesophageal and gastric lesions with the progression of mercuric chloride to the caecum, colon transverse, and the left colon. There was however a suspicion of mediastinitis.

During this period the patient had all of the blood investigations done which shows an inflammatory biological response but an overall stabilisation of her medical status/condition for which we were able to carry out another CT scan with injection and ingestion of contrast 22 days after her initial presentation. The scanner showed us evidence of oesophageal perforation and a broncho-oesophageal fistula. The colonic wall lining showed oedema but otherwise, there was not any suspicion of a perforated colon.

The patient was rushed to the operating theatre on the 23rd day where she had a total oesophago-gastrectomy with oesophagostomy and a jejunostomy for enteral alimentation. She also had two chest tubes placed due to the broncho-oesophageal fistula.

The period post-surgery was very difficult for this young patient who required a lot of assistance and enteral nutrition. She was cared for by a multidisciplinary team including surgeons, gastroenterologists, psychiatrists, psychologists, poison control center and a waste management team. The mercury level on the 59th day after admission dropped down to $248 \mathrm{ug} / \mathrm{L}$.

Meanwhile, a month later, as the patient was recovering from a heavy surgery she started to develop episodes of rectal haemorrhage requiring multiple blood transfusions, having had two CT scans the first showing an ischemic colonic lesion and the other showing rectal pneumatosis extending up to the sigmoid. She underwent a proctoscopy which did not show any mucosal changes, however, she was taken into surgery and had a laparotomy with a left lateral colostomy. We were confronted with a patient who had visible symptoms due to the changes to her colonic wall mucosa and a threat of going into haemorrhagic shock. This decision was taken wisely as we weighed the risk/benefit knowing the difficulty it will propose to us a challenge in the reconstruction of her digestive tube in the future.

The progression of mercuric chloride was slow but very destructive with the results of many surgical procedures, dialysis, blood transfusions, the dependency on enteral alimentation, the psychological impact and more importantly the difficulty in the future for this patient to undergo a coloplasty

This is where this article becomes of interest and discusses the surgical importance of patients after ingestion of inorganic mercury to avoid the slow progression and the opportunity to utilise their colon for a coloplasty in the future for they can lead a near to normal life.

\section{Literature review}

Toxicity from mercury depends on the composition of the compound as well as the quantity of mercury and even more importantly the route of administration. The different forms of mercury and their biological behaviour in the human body after ingestion is well explained by $\mathrm{NJ}$ and RE in their article 'toxicity of Mercury' [3].

They explain that inorganic mercury salts have been commonly used in teething powders, skin lightening creams and as preservatives in eye drops. These salts present a far greater hazard if ingested orally due to their greater water solubility in comparison with elemental mercury.

However, they are not lipid soluble and therefore cannot cross the blood-brain barrier. They concentrate mainly in the liver (excreted as bile) and kidneys (excreted as urine) [3].

The symptoms and signs arise in two phases, initially by a burning sensation of in the chest, discoloration of the mucous membrane and gastrointestinal pain along with vomiting and bloody diarrhoea risking a hypovolaemic shock. Secondly comes the stomatitis, loosening of teeth and renal damage from the accumulation of mercury in the proximal convoluted tubules [3,9]. Proximal tubular impairments have been documented by WANDS et al, as they have analysed the concentration of mercury in 2 post-mortem cases after chronic mercury poisoning due to abuse of laxatives. The two cases demonstrate tubular atrophy, and an ongoing renal damage was apparent as spotty areas of regenerating tubular epithelium, however, in one case it was more severe in the proximal tubule [10].

As there is not enough literature regarding this subject, [6], explained which may be the largest ingestion of elemental mercury which presented in a 33 year old lady with past medical history of depression, she ingested about 12.5 to $17.5 \mathrm{~kg}$ of mercury (equivalent to 2500 thermometers) and treated with sodium dimercaptopropanesulfonate $(0.25 \mathrm{mg} / \mathrm{dl}$ IM for three days followed by a three day rest, for a total of five weeks).

A 53-year-old chemistry teacher with a clinical history of generalised dystonia with cervical focal who had a rechargeable brain stimulator implanted years ago was found on her bedroom floor admitting she has ingested mercuric oxide. She developed hypokalemia from vomiting, initially her liver and renal function tests showed no change. Preventive measures were immediately carried out (gastric lavage, activated charcoal, KCL and fluids). Confirmation of caustic gastritis seen in the endoscopy and acute respiratory distress syndrome (ARDS) seen on a thoracic CT scan. Despite the prompt medical care given as well as the use of chelating agents and having a renal replacement continuous venovenous hemofiltration which was eventually changed to sustained low-efficiency dialysis (SLED) she passed away 15 days after admission with a diagnosis of acute renal failure and ARDS. The post mortem measurements proved the distribution of mercury to be a wide and very specific with its corrosive effect on the gastrointestinal tract shown by hepatic and acute tubular necrosis [4].

A 26-year-old lady ingested $0.9 \mathrm{~g}$ of mercuric chloride in a suicide attempt with endoscopic examination showing the necrotic effects in 
the oesophageal and gastric mucus. She was intubated due to dyspnea and laryngeal oedema and treated with dimercaprol (BAL) $400 \mathrm{mg}$ IM per day. She also underwent hemodialysis and plasma exchange with a full recovery four months later [5].

British anti-Lewiste, also known as dimercaprol, is a heavy metal chelating agent used to treat certain heavy metal poisoning cases. During World War II, BAL was used to reduce the risk of death for soldiers from Lewisite, a very powerful arsenic-based chemical warfare agent [11].

BAL is used nowadays for the treatment of inorganic and elemental mercuric salt poisoning.

Sodium dimercaptopropanesulfonate (DMSA) is a water soluble analogue of BAL has been proven by the WHO to be the best treatment of children with urinary mercury levels equal or greater than $50 \mu \mathrm{g} / \mathrm{mL}$ creatinine, if they are even asymptomatic [12].

Most of the articles concerning poisoning by inorganic mercury tend to prove medical, endoscopic and dialysis as the treatments used. However, Murphy et al described a case of a young male patient who presented to the emergency department 12 hours after poisoning with an unknown dose of mercuric chloride. Resuscitative measures (ventilation of lungs, haemodialysis and parenteral nutrition) as well as BAL was introduced immediately with a sequence of unsatisfactory results including a total colectomy due to massive gastrointestinal haemorrhage and toxic colon dilatation. Unfortunately, the postoperative phase included septicaemia and cerebellar abscess which were responsible for his death on day 47 [13]. A small amount of mercuric ions can cross the blood-brain barrier as explained by Murphy, et al. [13]).

For our patient, unfortunately, conservative treatment was not a success and we were obliged to operate in order to save her life. The dose she ingested and the manner in which it was ingested probably played an important role in the deterioration of the oesophageal, gastric and colonic mucus. If she was operated on immediately upon arrival, we could have prevented the progression of necrosis and the consequences leading to a colostomy.

The patient was followed regularly for a period of 2 years, a year and four months after her initial presentation she was rehospitalised for closure of her colostomy but as a topography was demanded before the surgical intervention we noticed a colonic stenosis $10 \mathrm{~cm}$ proximal to the colostomy which was initially investigated by a colonoscopy showing an inflammatory stenosis. The patient was taken to the operating theatre for closure and segmental colectomy with colonocolic anastomosis.

Meanwhile, her surgeon is seeing her on a regular basis to organise a coloplasty to be effectuated under better circumstances.

\section{Discussion}

In the mortality and morbidity meetings, this patient's file can bring up many exclamation marks, knowing the severity of mercuric chloride and its critical impact on the digestive tube we could have operated on her as soon as possible to restore the chance for a reconstructive intervention in order for her to lead a near to normal life. An urgent oesophagectomy in the next few hours after ingestion could protect from the systemic effects of the mercuric chloride and from caustic effects in both oesophagus and possibly the right colon.

Understanding the pharmacokinetics of this compound and all its forms is extremely necessary when dealing with a suicidal case even though it's a rare case yet the impact on this patient's life was very important and life-threatening. The approach is multidisciplinary, and an earlier surgical intervention should be considered immediately upon arrival.

This is why it is important to start the chelating agent immediately which has an affinity for inorganic mercury allowing it to be excreted rapidly and to have an endoscopic examination as soon as arrival if the patient state is stable. The initial problem may lay in the endoscopic investigation as the oesophageal and gastric lesions may be classified as not severe but can eventually deteriorate in a manner that can be lifethreatening and demanding an aggressive surgical intervention. Some articles suggest gastric lavage to be carried out immediately risking the high risk of aspiration [7].

Sanchez et al, clearly listed the therapeutic aim after inorganic mercury poisoning in 1963 :

- Immediate removal of poison by gastric lavage.

- Prevention of its toxicity with early administration of BAL

- Continuous use of BAL for 7 days or longer.

- Early and frequent haemodialysis to support the patient through the period of oliguria and possibly to remove the toxic mercury ion.

- Colectomy and removal of the rectum when haemorrhage from ulcerative colitis becomes a threat to life.

Their therapeutic criteria have been proven successful in many articles describing the conservative management of poisoning by lower doses of mercuric chloride with 'damage control colectomy' once haemorrhage appears in the later stages. For example, in the article by Murphy, et al. [13], their patient had undergone a total colectomy with a terminal ileostomy after an important colonic haemorrhage.

It's a lifesaving procedure but if this patient had presented earlier, he could have benefited from an oesophagectomy with or without gastrectomy and they could have probably avoided the corrosive consequences and his inevitable death.

Our case is an individual case most importantly as to how the patient decided to ingest the inorganic mercury compound but may be eligible to discuss the destructive pathway of this compound and predict the surgical intervention much earlier. The precocity of our endoscopic examination has failed our surgical instincts and has given us time for decision making before indulging in a very aggressive lifesaving surgery [14].

The oropharyngeal examination is critical, and its importance is integrated as it may result in difficult intubation and for our surgical intervention requiring an oesophagostomy.

\section{Conclusion}

Quantifying the dose, type of mercuric compound ingested and the way it reacts can help us predicate or narrow our choice to a better solution. Conservative Vs surgical intervention is a critical decision to make and is crucial for the patient. One being a very careful approach and not wanting to do further harm and the other is to correct the harm done and saving the gastrointestinal tract in order to reconstruct it in the future. For us, it became clearer as time passed that our late surgical intervention rendered the future of reconstructive surgery difficult but yet not impossible. 
This is a rare case study but mainly a lesson that teaches us the severity of poisoning by mercuric chloride ingestion and the earlier we intervene with an oesophagectomy the better chances the patients will have at survival by minimising the ongoing corrosive process of the gastrointestinal tract.

\section{Declarations}

Ethical statement: This is a case study and not a clinical trial or research, therefore it does not require a review by the ethical committee. The patients' identity has been respected and kept anonymous throughout the article. The author 'myself' and corresponding authors take full responsibility in the case of any breach of confidentiality and ethics. The article is in compliance with the ethical standards.

\section{Informed consent of publication}

Received from the patient (oral and written)

\section{Availability of data and material}

N/A

\section{Funding}

No funding has been received for this article

\section{Competing interests}

Pr. Monneuse, Dr. Gruner and I declare that there is no conflict of interest

The authors declare that they have no competing interests

\section{Authors' contribution}

- SA: general surgeon post residency who gathered all the information and patients file. Also participated in studying the case, doing literature review and writing the article.

- LG: Operated on the patient and helped provide the documented details of the patients' surgeries and consultations in order to integrate them in the article.
- OM: Participated in the emergency care of the patient and helped with analysis and approval of the article before sending it for submission.

\section{Acknowledgement}

Not applicable.

\section{References}

1. Rice KM, Walker EM, Wu M, Gillette C, Blough ER (2014) Environmental Mercury and Its Toxic Effects. J Prev Med Public Health 47: 74-83. [Crossref]

2. Poupon J (2007) L'exposition au mercure en 2007 toxicité et prise en charge. Revue Francophone des Laboratoires 390: 51-56.

3. Langford NJ, Ferner RE (1999) Toxicity of Mercury. Journal of human hypertension 13: 651-656.

4. Dias D, Bessa J, Guimarães S, Soares ME, Bastos MDL, et al. (2016) Inorganic mercury intoxication: A case report. Forensic Sci Int 259: e20-e24. [Crossref]

5. Yoshida M, Satoh H, Igarashi M, Akashi K, Yamamura Y, et al. (1997) Acute Mercury Poisoning by Intentional Ingestion of Mercuric Chloride. Tohoku J Exp Med 182: 347 352. [Crossref]

6. Song Y, Li A (2007) Massive elemental mercury ingestion. Clin Toxicol 45: 193 [Crossref]

7. Zag L, Berkes G, Takács IF, Szepes A, Szabó I (2017) Endoscopic management of massive mercury ingestion. Medicine 96: e6937. [Crossref]

8. Ollier E, Carcel C, Bost M, Grenet G, Billon A, et al. (2017) Intoxication sévère au chlorure de mercure. Toxicologie Analytique et Clinique 29: S42.

9. Park JD, Zheng W (2012) Human Exposure and Health Effects of Inorganic and Elemental Mercury. J Prev Med Public Health 45: 344-352. [Crossref]

10. Wands JR, Weiss SW, Yardley JH, Maddrey WC (1974) Chronic inorganic mercurypoisoning due to laxative abuse-clinical and ultrastructural study. Am J Med 57: 92-101. [Crossref]

11. Vilensky JA, Redman K (2003) British anti-Lewisite (dimercaprol): an amazing history. Ann Emerg Med 41: 378-383. [Crossref]

12. Forman J, Moline J, Cernichiari E, Sayegh S, Torres JC, et al. (2000) A cluster of pediatric metallic mercury exposure cases treated with meso-2,3-dimercaptosuccinic acid (DMSA). Environ Health Perspect 108: 575-577. [Crossref]

13. Murphy MJ, Culliford EJ, Parsons V (1979) A case of poisoning with mercuric chloride Resuscitation 7: 35-44. [Crossref]

14. Sanchez-Sicilia L, Seto DS, Nakamoto S, Kolff WJ (1963) Acute Mercurial Intoxication Treated by Hemodialysis. Ann Intern Med 59: 692-706. [Crossref]

Copyright: (C2020 Al Awad S. This is an open-access article distributed under the terms of the Creative Commons Attribution License, which permits unrestricted use, distribution, and reproduction in any medium, provided the original author and source are credited. 